\title{
The Prevalence of Iron Deficiency Anemia and its Associated Risk Factors among a Sample of Females in Riyadh, Saudi Arabia
}

\author{
Abdullah Mansour Alswailem, Sulaiman Mohammed Alahmad, Muath Ali Alshehri \\ Almareefa colleges \\ Corresponding author: Abdullah Mansour Alswailem, Email: Abdullah.m.alswailem@gmail.com, mobile no: 0544435539
}

\begin{abstract}
Objectives: To assess the prevalence of iron deficient anemia (IDA) within Saudi Arabian females and to examine the risk factors leading to the IDA.

Methods: A cross-sectional study on 683 healthy females aged between 18 and 40 years was performed within April 2016. Data on the participants' socio-demographics, diet, health, anthropometry, and hematological and biochemical iron status indices were gathered. Univariate and multivariate logistic regression analysis were used in order to reveal the IDA risk factors.

Results: A total of 683 non-pregnant women aged between 18-40 years were included in the analysis. The prevalence of IDA was $41.6 \%$. In the multivariate regression analysis; inadequate iron and vitamin C intakes, infrequent ( $\leq 2$ times per week) consumption of red meat and fish, menstruation disorder eg: (Twice or more per month), (more than 8 days), (blood clotting and heavy blood flow),blood disorder and previous blood transfusion, past personal history of IDA and familial history of IDA were significantly associated with increased odds of IDA.

Conclusion: To reduce IDA in Saudi Arabian women, the country needs a multifaceted approach that incorporates iron supplementation, food fortification, rich dietary iron sources and by raising awareness of the food and drinks which facilitate or hinder the bioavailability of iron.
\end{abstract}

Keywords: Iron deficiency anemia, women, prevalence, food, risk factors.

\section{INTRODUCTION}

Anemia is recognized as the world's most widespread nutritional disorder, affecting 1.6 billion people that constitute about $25 \%$ of the global population $^{(1)}$. Iron deficiency (ID) is a state in which there is insufficient iron to maintain the normal physiological function of tissues ${ }^{(2)}$. IDA is the most frequent cause of anemia world-wide and is a very common disorder in daily medical practice ${ }^{(3)}$. Iron deficiency anemia is the most common nutritional disorder seen all over the world, more in the developing countries, particularly, affecting young children of 6-24 months of age, adolescents, women of reproductive age group and pregnant/ lactating women ${ }^{(4,5)}$.

Anemia is associated with adverse physiological and psychological outcomes in women especially pregnant and nursing mothers which affect also the babies. For the mother, these include cardiovascular problems, reduced physical activity and cognitive performance, reduced immune function, tiredness, and increased depressive episodes, while, for the infant, these include preterm birth, fetal growth restriction, intrauterine fetal death, and neonatal infection ${ }^{(6-9)}$.

The main cause of deficiency is consumption of cereal-based diets, which provide non-haem iron of poor bioavailability ${ }^{(10)}$, prolonged negative imbalance between a person's dietary intake of iron and their body's physiological demand ${ }^{(11,12)}$ and Deficiency of other nutrients such as vitamins A, C, B2, B12 and folic acid may also cause anemia ${ }^{(13)}$. Other identified non-nutritional causes of anemia include malaria, hemodialysis patients, hookworm infestation, chronic infection and inflammation, and hemoglobin opathies such as thalassemia ${ }^{(13-17)}$.

Our study aimed at exploring the risk factors of ID in Saudi Arabian females, to design an effective intervention program that can target the underlying causes and prevent or treat anemia in this population group. It is of paramount importance to identify the potential risk factors for anemia and ID. Therefore, the present study was designed to identify the potential risk factors associated with iron deficiency anemia among females in kingdom of Saudi Arabia.

\section{PATIENTS AND METHODS}

\section{Study design}

This cross-sectional observational study was performed during April 2018 with a sample of 683 healthy women aged between 18 and 40 years, who were from Riyadh city, Saudi Arabia.

\section{Study population}

The study sample excluded females who were currently pregnant or breastfeeding, those who were taking medication or nutritional supplement. All the participants provided written informed consent 
acknowledging the investigation's purpose and were assured of the confidentiality of the results.

\section{Questionnaire study}

The questionnaire covered 5 main topics: the participants' personal socio-demographic data (eg, their age, marital status, and monthly household income); dietary information relating to their intakes of iron-rich foods and of iron absorption-inhibiting or enhancing foods, and whether or not they followed particular dietary regimens; their obstetric and gynecological history (ie, their menstrual history, including its frequency and duration; their usual menstrual flow during their cycle; whether or not they encountered blood clotting; their use of oral and other contraceptives, and if so, how long they had done so; and their number of children and the birth intervals between them); any current or past diagnoses of medical conditions (eg, chronic diseases, blood disorders, or blood transfusions); their history of smoking (if any); and, finally, their personal and family histories of IDA.

\section{Statistical Analysis}

The collected data were analyzed using the SPSS for Windows program (version 23.0, SPSS, Chicago, IL, USA).Statistical significance was set at $\mathrm{P}<.05$. A univariate logistic regression analysis was chosen as the screening method to assess the relationships between the respective putative predictors and the outcome variable (anemia), which were categorized into either anemic or non-anemic. The predictor variables identified as significant by the univariate logistic analysis were then entered into a multiple logisticregression model to establish which of them could most accurately predict occurrences of IDA among the study's sample ofparticipants. The odds ratios (ORs) and their $95 \%$ confidenceintervals (CIs) for each of the variables were then generated to clarify the respective association of each of the risk factors withthe participants' anemia status

\section{Administrative consideration:}

The Researchers fulfilled all the required officialapprovals.

\section{Ethical Considerations:}

Ethical approval has been received from the ethical committee. Before interviewing, the researchers explained the purpose to all respondents and oral consent was collected from all the participants. The participants had the right not to participate in the study or to withdraw from the study prior to completion. Confidentiality and privacy were guaranteed for all participants.

\section{RESULTS}

\section{Recruitments}

\section{Patient baseline characteristics}

683 female of Saudi nationality were enrolled in Riyadh Unit of Hematology - Department of Internal Medicine consented to participate in this research. Their ages ranged between 18 and 40 years, with a mode age between 31-40 years. The study population's overall prevalence of IDA was found to be $41.6 \%$ (Figure 1).

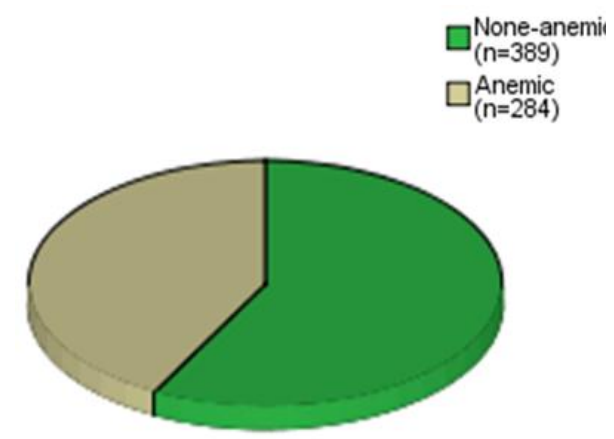

Figure (1): Prevalence of iron deficiency anemia (IDA) among the study sample of female in Riyadh city.10 participants with missing data.

Table 1 shows the unadjusted ORs for the factors associated with IDA among the research sample of Saudi Arabian females. The univariate analysis found that citrus consumption ( $\mathrm{OR}=2.3 ; 95 \%$ CI:0.7-7.1), infrequent ( $\leq 2$ times per week) red meat consumption $(\mathrm{OR}=6.1 ; 95 \% \mathrm{CI}$ : 1.3-28.7), poultry $(\mathrm{OR}=1.4 ;$; $95 \% \mathrm{CI}: 0.4-4.9)$, fish (OR 1.9=; 95\% CI:0.9-4.1), regime (OR =1.5; 95\%CI:0.3-8.2), smoke (OR=1.1; 95\%CI:0.6 -1.8), personal histories of IDA (OR $=1.1 ; 95 \%$ CI: 0.1-12.2), and family histories of IDA (OR $=3.2$; 95\% CI: 1.3-7.7) were each significantly associated with an increased risk of IDA for the participating females. The only factors which emerged as statistically significant from the adjusted logistic regression analysis model (Table 2) were insufficient intakes of meat $(\mathrm{OR}=1.1 ; 95 \% \mathrm{CI}$ : 0.7-1.7), fish 5.8(OR $=5.8 ; 95 \% \mathrm{CI}: 1.3-25.7)$ and vitamin $\mathrm{C}(\mathrm{OR}=1.3 ; 95 \% \mathrm{CI}: 0.9-2.1)$, increased menstruation frequency (twice or more per month) $1.7(\mathrm{OR}=1.7 ; 95 \% \mathrm{CI}: 0.7-4.1)$, Blood clotting during menstruation2(OR $=2$; 95\% CI: 0.3-11.4), blood disorder $(\mathrm{OR}=2.6 ; 95 \% \mathrm{CI}: 1.5-4.5)$ and the possession of family history of IDA (OR $=3.2 ; 95 \%$ CI: 1.3-7.7). 
Table (1): Univariate logistic regression analysis of the factors associated with iron deficiency anemia among the study sample of females in Riyadh city.

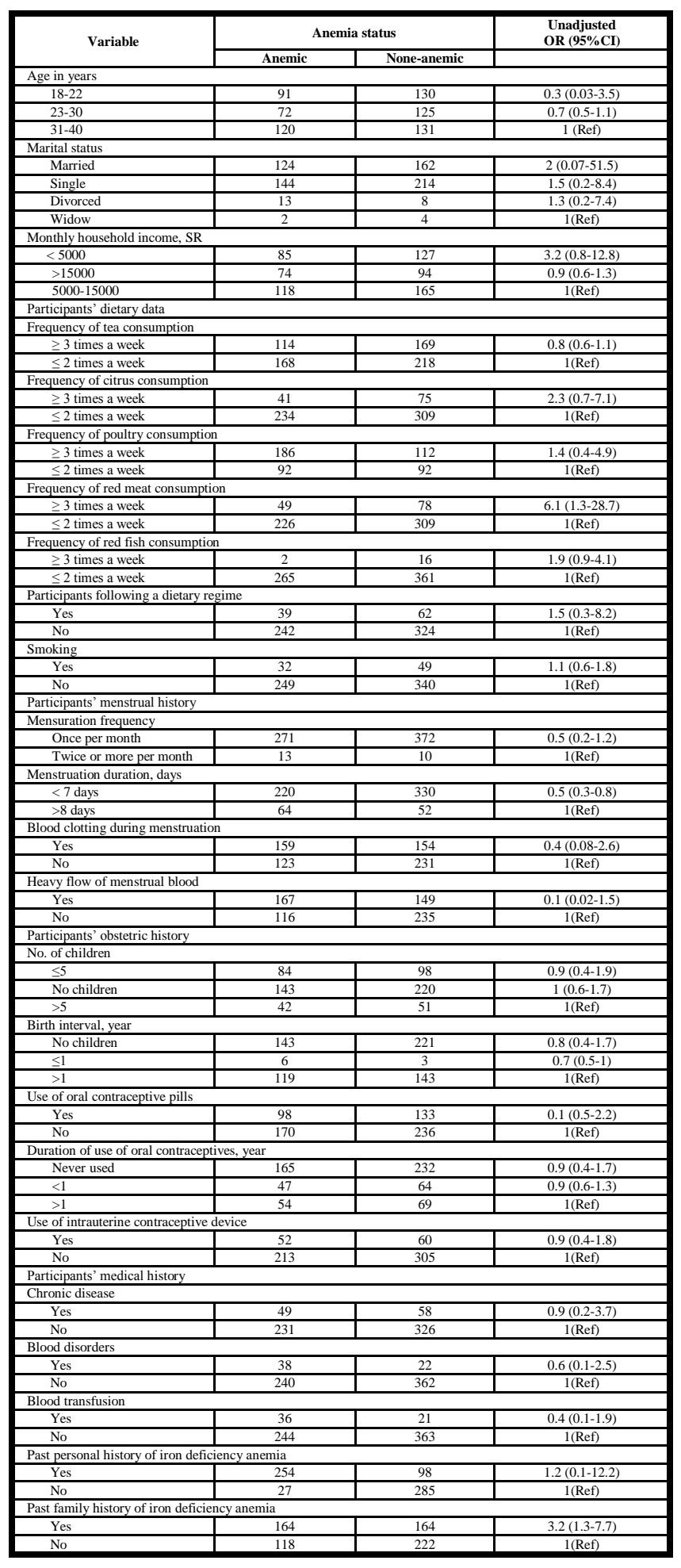

Abbreviations: CI, confidence interval; OR, odds ratio; Ref., reference; SR, Saudi riyal.
2 missing data in marital status, 3 missing data in smoking, 4 missing data in age and frequency of tea consumption, 10 missing data in total income, 14 subjects with missing data in citrus consumption, 11 subjects with missing data in poultry, meat consumption and blood disorder, 29 subjects with missing data in fish consumption, 6 subjects with missing data in diet regime, blood clotting and heavy blood flow during menstruation, 7 subjects with missing data in menstruation frequency and days,35 subjects with missing data in no of children, 38 subjects with missing data in birth interval, 36 subjects with missing data in contraceptive pills and 42 missed subjects in its duration, 43 subjects with missing data in IU contraceptive, 9 subjects with missing data in chronic disease, received blood and personal history of IDA,11 subjects with missing data in blood disorder and 5 subjects with missing data in family history of IDA.

Table (2): Multivariate logistic regression analysis of the factors associated with iron deficiency anemia among the study sample of femalesin Riyadh city.

\begin{tabular}{|c|c|}
\hline Variable & $\begin{array}{c}\text { Unadjusted } \\
\text { OR }(95 \% \mathrm{CI}) \\
\end{array}$ \\
\hline \multicolumn{2}{|l|}{ Intake of citrus } \\
\hline$<$ Recommended intake & $1.3(0.9-2.1)$ \\
\hline$\geq$ Recommended intake & 1 (Ref) \\
\hline \multicolumn{2}{|c|}{ Frequency of red meat consumption } \\
\hline$<$ Recommended intake & $1.1(0.7-1.7)$ \\
\hline$\geq$ Recommended intake & 1 (Ref) \\
\hline \multicolumn{2}{|c|}{ Frequency of fish consumption } \\
\hline$<$ Recommended intake & $5.8(1.3-25.7)$ \\
\hline$\geq$ Recommended intake & 1 (Ref) \\
\hline \multicolumn{2}{|l|}{ Mensuration frequency } \\
\hline$>$ normal frequency & $1.7(0.7-4.1)$ \\
\hline$\leq$ normal frequency & 1 (Ref) \\
\hline \multicolumn{2}{|l|}{ Menstruation duration, days } \\
\hline$>$ normal days & $1.8(1.2-2.7)$ \\
\hline$\leq$ normal days & 1 (Ref) \\
\hline \multicolumn{2}{|c|}{ Blood clotting during menstruation } \\
\hline Yes & $2(0.3-11.4)$ \\
\hline No & 1 (Ref) \\
\hline \multicolumn{2}{|l|}{ Blood disorders } \\
\hline Yes & $2.6(1.5-4.5)$ \\
\hline No & 1 (Ref) \\
\hline \multicolumn{2}{|l|}{ Blood transfusion } \\
\hline Yes & $2.5(1.4-4.4)$ \\
\hline No & 1 (Ref) \\
\hline \multicolumn{2}{|c|}{ Past personal history of iron deficiency anemia } \\
\hline Yes & $3.3(0.6-16.1)$ \\
\hline No & 1 (Ref) \\
\hline \multicolumn{2}{|c|}{ Past family history of iron deficiency anemia } \\
\hline Yes & $1.8(1.3-2.5)$ \\
\hline No & 1 (Ref) \\
\hline
\end{tabular}

Abbreviations: CI, confidence interval; OR, odds ratio; Ref., reference. 


\section{DISCUSSION}

Anemia is not a specific entity but an indication of an underlying pathologic process or disease. WHO lists iron deficiency (ID) as one of "Top Ten Risk Factors contributing to death" ${ }^{(18)}$. For this reason, the present study focused on establishing the prevalence of IDA among females in Riyadh city in Kingdom of Saudi Arabia and identifying the risk factors associated with that condition. The study proved an overall IDA prevalence of $41.6 \%$, which is lower than the prevalence of IDA in south Asian countries such as Bangladesh and Pakistan which reached $55 \%{ }^{(19)}$. Also, significantly lower rate than that found by a prior study among a sample of female Saudi university students, which reported aprevalence of IDA of $64 \%{ }^{(20)}$. In contrast, these results are significantly higher compared to those collected through a study conducted in Saudi Arabia with an overall IDA prevalence of $12.5 \%{ }^{(21)}$. In China the reduction of IDA prevalence is especially remarkable i.e., the prevalence was halved from $20 \%$ to the current level of $8 \%$ within a decade ${ }^{(22-24)}$.

Our results have indicated that most of the females in the study sample were anemic and reported inadequate intakes of citrus, along with a lower level of consumption ( $\leq 2$ times per week) of red meat and fish. These factors were found to be associated with a statistically significant increased risk of IDA. Eighty-seven patients and 203 controls were enrolled in the study. Low frequency of eating meat, vegetables or drinking juices right with vitamin $\mathrm{C}$ increased the risk of having iron deficiency anemia by 2-4 fold (odds ratio $=2.06$, $95 \%$ confidence interval $1.20-3.54)^{(25)}$. This result is in compliance with that of a study conducted in Vietnam which concluded that eating $<1$ serving of meat/ week, and farming were significantly associated with anemia in women and children ${ }^{(26)}$. Also, in Brazil a research assured that two foods(meat and beans) are responsible for the totality of this mineral in the habitual Brazilian diet registering a decreasing intake in the last decades which is considered the main leading cause of IDA (27). Inadequate intakes of vegetables, fruits, dairy, soybean, eggs, fish and excessive intakes of cereals, meat, cooking oil, salt were common in middle aged and elderly population in southwest China which may increase the risk of anemia ${ }^{(28)}$.

Our study has an advantage rather than other studies conducted in KSA as the increased number of sample population and wider age scale (from 18-40 years) justified our results which could be considered more representable to the prevalence of IDA between females and its risk factors. However, the study was not free from limitations as a cross sectional study has no causal associations which could be established between theinfluencing factors and personal IDA and also the participants who formed the study sample were all volunteers within Riyadh city, therefore, they may not be freely chosen to represent the wider female population in Saudi Arabia.

\section{CONCLUSION}

This study confirmed a prevalence of IDA among its sampleof healthy young Saudi female aged from $18-40$ years Riyadh city of $41.6 \%$. The study also reported that the main risk factors related were fish consumption, problem with menstruation like increased frequency, days and heavy blood flow and a past personal and familial history of IDA. The findings presented addressed that dietary iron deficiency has to receive special attention through iron supplementation programs targeting women and adolescent girls. Saudi females are in great need for receiving intensive educational awareness on specific strategies designed to improve nutritional habitsby encouraging the consumption of rich dietary sources of iron (eg, red meat), fish as bioavailability well as vitamin C-rich foods and decrease consumption of tea and coffee which alter iron. Understanding risk factors which induce anemia support lower prevalence of IDA, thus, leading to greater impact on hemoglobin levels and lessen side effects resulted from these diseases.

\section{REFERENCES}

1. Foote EM, Sullivan KM, Ruth LJ, Oremo J, Sadumah I, Williams TN, Suchdev PS (2013): Determinants of anemia among preschool children in rural, western Kenya. The American journal of tropical medicine and hygiene,88(4):757-64.

2. Benoist BD, McLean E, Egll I, Cogswell M (2008): Worldwide prevalence of anaemia 19932005: WHO global database on anaemia. Worldwide prevalence of anaemia 1993-2005: WHO global database on anaemia. https:// www. cabdirect. org/ cabdirect/abstract/20093013528

3. Estadella J, Villamarín L, Feliu A, Perelló J, Calaf J (2018): Characterization of the population with severe iron deficiency anemia at risk of requiring intravenous iron supplementation. European Journal of Obstetrics \& Gynecology and Reproductive Biology,224:41-4. 
4. Lokeshwar MR, Mehta M, Mehta N, Shelke P, Babar N (2011): Prevention of iron deficiency anemia (IDA): How far have we reached?. The Indian Journal of Pediatrics, 78(5):593-602.

5. Abu-Ouf NM, Jan MM (2015): The impact of maternal iron deficiency and iron deficiency anemia on child's health. Saudi medical journal,36(2):146.

6. Froessler B, Gajic T, Dekker G, Hodyl NA (2018): Treatment of iron deficiency and iron deficiency anemia with intravenous ferric carboxymaltose in pregnancy. Archives of gynecology and obstetrics, 8:1-8.

7. Haider BA, Olofin I, Wang M, Spiegelman D, Ezzati M, Fawzi WW (2013): Anaemia, prenatal iron use, and risk of adverse pregnancy outcomes: systematic review and meta-analysis. Bmj.,346:f3443.

8. Congdon EL, Westerlund A, Algarin CR, Peirano PD, Gregas M, Lozoff B, Nelson CA (2012): Iron deficiency in infancy is associated with altered neural correlates of recognition memory at 10 years. The Journal of pediatrics, 160(6):1027-33.

9. Erez Azulay C, Pariente G, Shoham-Vardi I, Kessous R, Sergienko R, Sheiner E (2015): Maternal anemia during pregnancy and subsequent risk for cardiovascular disease. The Journal of Maternal-Fetal \& Neonatal Medicine,28(15):1762-5.

10. Zimmermann MB, Hurrell RF (2007): Nutritional iron deficiency. The Lancet, 370(9586):511-20.

11. Abbaspour N, Hurrell R, Kelishadi $\mathbf{R}$ (2014): Review on iron and its importance for human health. Journal of research in medical sciences: the official journal of Isfahan University of Medical Sciences, 19(2):164.

12. Hurrell R, Egli I (2010): Iron bioavailability and dietary reference values-. The American journal of clinical nutrition,91(5):1461S-7S.

13. Ahmed F, Al-Sumaie MA (2011): Risk factors associated with anemia and iron deficiency among Kuwaiti pregnant women. International journal of food sciences and nutrition, 62(6):585-92.

14. Stoltzfus RJ, Albonico M, Chwaya HM, Savioli L, Tielsch J, Schulze K, Yip R (1996): Hemoquant determination of hookworm-related blood loss and its role in iron deficiency in African children. The American journal of tropical medicine and hygiene,55(4):399-404.
15. Madore F, et al. (1997): Anemia in hemodialysis patients: variables affecting this outcome predictor. Journal of the American Society of Nephrology,8(12): p. 1921-1929.

16. Dreyfuss ML, Stoltzfus RJ, Shrestha JB, Pradhan EK, LeClerq SC, Khatry SK, Shrestha SR, Katz J, Albonico M, West Jr KP (2000): Hookworms, malaria and vitamin A deficiency contribute to anemia and iron deficiency among pregnant women in the plains of Nepal. The Journal of nutrition, 130(10):2527-36.

17. Semba RD, Bloem MW (2002): The anemia of vitamin A deficiency: epidemiology and pathogenesis. European journal of clinical nutrition,56(4):271.

18. Jarrah SS, Halabi JO, Bond AE, Abegglen J (2007): Iron deficiency anemia (IDA) perceptions and dietary iron intake among young women and pregnant women in Jordan. Journal of Transcultural Nursing,18(1):19-27.

19. Gillespie S (1997): Malnutrition in south Asia: a regional profile. https:// www. popline. org/ node/ 278829

20. Al Hassan NN (2015): The prevalence of iron deficiency anemia in a Saudi University female students. Journal of Microscopy and Ultrastructure,3(1):25-8.

21. Alzaheb RA, Al-Amer O (2017): The Prevalence of Iron Deficiency Anemia and its Associated Risk Factors Among a Sample of Female University Students in Tabuk, Saudi Arabia. Clinical Medicine Insights: Women's Health, 10:1179562X17745088.

22. Sinha N, Deshmukh P, Garg B (2008): Epidemiological correlates of nutritional anemia among children (6-35 months) in rural Wardha, Central India. Indian journal of medical sciences,62(2):45.

23. Dallman PR, Yip R, Oski FA(1993): Iron deficiency and related nutritional anemias. Hematology of infancy and childhood,4:413-50.

24. Lokeshwar MR, Mehta M, Mehta N, Shelke P, Babar N (2011): Prevention of iron deficiency anemia (IDA): How far have we reached? The Indian Journal of Pediatrics,78(5):593-602.

25. Al-Quaiz JM(2011): Iron deficiency anemia. A study of risk factors. Saudi medical journal,22(6):490-6. 